\title{
Relationship between coronary artery calcification and myocardial ischemia on computed tomography myocardial perfusion in patients with stable chest pain
}

\author{
Mohammed El Mahdiui, MD, ${ }^{\mathrm{a}}$ Jeff $M$. Smit, $\mathrm{MD},{ }^{\mathrm{a}}$ \\ Alexander R. van Rosendael, MD, ${ }^{a}$ J. Wouter Jukema, $M D, P h D,{ }^{a}$ \\ Jeroen J. Bax, MD, PhD, a and Arthur J. H. A. Scholte, MD, PhD ${ }^{a}$ \\ a Department of Cardiology, Heart Lung Center, Leiden University Medical Center, Leiden, \\ The Netherlands
}

Received Jul 8, 2019; accepted Aug 14, 2019

doi: $10.1007 / \mathrm{s} 12350-019-01869-8$

Background. Coronary artery calcium (CAC) score has shown to provide incremental prognostic information when added to the Framingham risk score. Although the relation between CAC and myocardial ischemia has been evaluated, there has been little evaluation of the relationship between CAC score and inducible myocardial ischemia on computed tomography myocardial perfusion (CTP).

Methods and Results. Patients who were referred with stable chest pain from the outpatient clinic and who underwent non-contrast computed tomography scan, coronary computed tomography angiography, and adenosine stress CTP were included in this study. CAC score was subdivided in four groups ( 1 to $99 ; 100$ to 399,400 to 999, and $\geq 1000$ ). Inducible myocardial ischemia was considered when reversible perfusion defects were observed in $\geq 1$ segment. A total of 131 patients (age $62 \pm 9.4$ years; $56 \%$ male) were included. The median CAC score was 241 (73 to 539). Forty-nine patients (37\%) had evidence of inducible myocardial ischemia. The presence of inducible myocardial ischemia increased with increasing CAC score from $22 \%$ in the CAC score 1 to 99 subgroup to 35,47 , and $65 \%$ in the 100 to 399,400 to 999 , and $\geq 1000$ CAC score subgroup, respectively. In multivariable analysis CAC score was the only determinant that significantly predicted the presence of inducible myocardial ischemia on CTP.

Conclusions. In a population of symptomatic patients, the majority of patients with extensive calcification had evidence of inducible myocardial ischemia on CTP. CAC score was the only independent predictor of inducible myocardial ischemia on CTP. (J Nucl Cardiol 2021;28:170714.)

Key Words: CAD $\cdot$ myocardial ischemia and infarction $\cdot$ CT Atherosclerosis

Electronic supplementary material The online version of this article (https://doi.org/10.1007/s12350-019-01869-8) contains supplementary material, which is available to authorized users.

The authors of this article have provided a PowerPoint file, available for download at SpringerLink, which summarises the contents of the paper and is free for re-use at meetings and presentations. Search for the article DOI on SpringerLink.com.
Reprint requests: Arthur J. H. A. Scholte, MD, PhD, Department of Cardiology, Heart Lung Center, Leiden University Medical Center, Albinusdreef 2, 2300 RC Leiden, The Netherlands; A.J.H.A.Scholte@lumc.nl 1071-3581/\$34.00

Copyright (C) 2019 The Author(s) 


\begin{tabular}{|ll|}
\hline Abbreviations \\
CAC & Coronary artery calcium \\
CAD & Coronary artery disease \\
CT & Computed tomography \\
CTA & Computed tomography angiography \\
CTP & Computed tomography myocardial \\
& perfusion \\
MPI & Myocardial perfusion imaging \\
SDS & Summed difference score \\
SPECT & Single-photon emission computed \\
& tomography \\
SRS & Summed rest score \\
SSS & Summed stress score \\
\hline \hline
\end{tabular}

See related editorial, pp. 1715-1717 likelihood of $\mathrm{CAD}^{21}$ The imaging protocol design at our center has been reported before. ${ }^{22,23}$ Patients with $\geq 1$ uninterpretable myocardial segments on CTP were excluded from analysis. Patients with a history of myocardial infarction or revascularization were also excluded from analysis. Contraindications for cardiac CT were atrial fibrillation, renal insufficiency, second or third degree atrioventricular block, known allergy to iodine-containing contrast agents, and pregnancy. Clinical data were prospectively entered into the departmental cardiology information system (EPD-Vision $($, Leiden University Medical Center, The Netherlands). The Dutch Central Committee on Human-related Research allows the use of anonymous patient data without previous approval of an institutional review board, provided that the data are acquired for routine patient care. All data used for this study were acquired for clinical purposes.

\section{Cardiac CT Acquisition}

Non-contrast CT, coronary CTA, and CTP were acquired on the same day, using a 320-row volumetric scanner (from 2013 until November 2015 Aquilion ONE, Canon Medical Systems, Otawara, Japan and from November 2015 the Aquilion ONE Genesis Edition, Canon Medical Systems, Otawara, Japan).

Patients were instructed not to consume caffeine products 24 hour before examination since CTP with adenosine might be performed. On the day of examination patients were evaluated $1 \mathrm{~h}$ prior to CT acquisition, by measuring the patient's heart rate and blood pressure. Metoprolol, $25 \mathrm{mg}$ up until $150 \mathrm{mg}$, was administrated orally if a patient's heart rate exceeded 60 beats per minutes (bpm) and no contraindications were present. Additional metoprolol could be administrated intravenously if the heart rate remained above $60 \mathrm{bpm}$ during scout images.

First, a low dose non-contrast enhanced scan was performed to determine the CAC score. Nitro-glycerine $(0.4 \mathrm{mg})$ was sprayed sublingual prior to coronary CTA. The coronary CTA was performed with the following scan parameters: detector collimation of $320 \times 0.5 \mathrm{~mm}, 350 \mathrm{~ms}$ gantry rotation time and temporal resolution of $175 \mathrm{~ms}$ for the Aquilion ONE and $275 \mathrm{~ms}$ gantry rotation time and temporal resolution of $137 \mathrm{~ms}$ for the Aquilion ONE Genesis Edition. Peak tube voltage was between 100 and $135 \mathrm{kV}$ and tube current between 140 and $580 \mathrm{~mA}$, depending on body mass index. The contrast agent (Iomeron 400, Bracco, Milan, Italy) was injected in the antecubital vein. First, 50 to $90 \mathrm{~mL}$ (depending on patient weight) contrast agent (flow rate 5 to $6 \mathrm{~mL} / \mathrm{s}$ ) was administrated, followed by $20 \mathrm{~mL}$ of a 1:1 mixture of contrast and saline and finally $25 \mathrm{~mL}$ of saline (flow rate $3 \mathrm{~mL} /$ s). Prospective ECG triggering was used to scan 70 to $80 \%$ of the RR-interval, in patients with heart rate $>65 \mathrm{pm}, 30$ to $80 \%$ of the RR-interval was covered. Real-time bolus tracking was performed in the descending aorta, and coronary CTA was performed the next beat when the threshold of 300 Hounsfield units (HU) was reached.

Stress CTP was performed at least 20 minutes after coronary CTA to achieve adequate myocardial contrast washout. After 4 minutes of continuous adenosine infusion $(0.14$ $\mathrm{mg} / \mathrm{kg} / \mathrm{min}$ ) and continuous electrocardiogram and blood pressure monitoring, contrast agent was given. After reaching 
the target threshold of $300 \mathrm{HU}$ in the descending aorta, CTP images were acquired the next heart beat scanning 80 to $99 \%$ of the RR-interval. The tube settings, injection protocol, and contrast agent were similar to the coronary CTA acquisition. If side-effects occurred during adenosine infusion, the administration was discontinued which resolved the side-effects rapidly and theophylline or atropine could be administrated if needed. The effective radiation dose was calculated by multiplying the dose-length-product by a conversion coefficient $0.014 \mathrm{mSv} /(\mathrm{mGy} \times \mathrm{cm}){ }^{24}$

\section{Image Reconstruction and Analysis}

Collected images were transferred to a workstation and analyzed using dedicated post-processing software (Vitrea FX 6.5; Vital Images, Minnetonka, Minnesota). For the assessment of the CAC score, images with a $3 \mathrm{~mm}$ slice thickness were reconstructed from the non-contrast $\mathrm{CT}$. To analyze the CAC score, pixels exceeding $130 \mathrm{HU}$ were recognized and encircled in the course of a coronary artery and calculated according to the Agatston method. ${ }^{1}$ CAC score was categorized into 4 subgroups, minimal to mild calcification $(\mathrm{CAC}$ score $=1$ to 99), moderate calcification (CAC score $=100$ to 399), severe calcification (CAC score $=400$ to 999 ) and extensive calcification (CAC score $\geq 1000$ ). For myocardial perfusion analysis, cardiac phases were reconstructed every $2 \%$ of the scanned interval. The phase with the best image quality was selected and interpreted with a narrow window width and level setting (W300/L150), according to the standard 17 myocardial segment model. ${ }^{25}$ For per vessel analysis, individual myocardial segments were assigned to the 3 major coronary arteries using also the standard 17 myocardial segment model. After the initial analysis, observers were allowed to adjust the display settings. All images were analyzed and interpreted by two trained observers. For the present analysis CTA images were exclusively used for rest perfusion data and not for stenosis degree and/or plaques analysis. For stress data, CTP images during adenosine infusion were used. CTP images were arranged in the short axis, vertical long axis and horizontal long axis with a slice thickness of $3 \mathrm{~mm}$. Each segment was scored for perfusion defects and if present, other phases were checked to differentiate between real perfusion defects or artifacts. $^{26}$ When perfusion defects were observed in $\geq 1$ segment, the CTP was considered abnormal. Summed difference score (SDS) was calculated by subtracting the summed rest score (SRS) from the summed stress score (SSS). To calculate the SRS and the SSS, all abnormal segments were added from the rest data and stress data, respectively. Inducible myocardial ischemia was defined by a $\mathrm{SDS} \geq 1$.

\section{Statistical Analysis}

Continuous variables are depicted as mean \pm standard deviation when normally distributed and median with 25 to 75 th percentile (interquartile range (IQR)) when non-normally distributed. Normally distributed variables were analyzed using the independent sample $t$ test and non-normally distributed variables using the Mann Whitney $U$ test or the
Kruskal Wallis. Summed scores are depicted as mean and range. Categorical variables are depicted as percentages and numbers and analyzed using the $\chi^{2}$ test. Correlation between CAC score as a continuous variable and extent of myocardial ischemia as assessed by SDS was tested with the Spearman correlation coefficient. Univariable and multivariable analysis were performed to evaluate the variables that were significantly associated with myocardial ischemia on CTP. Variables with a $P$ value $<0.1$ in univariable analysis and age and gender were included in the multivariable analysis. A $P$ value $<0.05$ was considered statistically significant. All statistical analyses were performed using SPSS version 23.0 (SPSS, Armonk, NY).

\section{RESULTS}

\section{Clinical Characteristics}

A total of 146 patients were identified. One patient was revascularized and was excluded from further analysis. We excluded 14 patients because of 1 or more uninterpretable myocardial segments on CTP, leaving 131 patients (age $62 \pm 9.4$ years; 56\% male) for analysis. Clinical characteristics are shown in Table 1. Prevalence of cardiovascular risk factors was high in the total population. There were no differences between both groups regarding the presence of cardiovascular risk factors, pre-test likelihood, or medication use.

\section{Coronary Artery Calcium Score}

The median CAC score of the study population was 241 (73 to 539). $41(31 \%)$ patients had minimal to mild calcification (CAC score 1 to 99), 43 (33\%) had moderate calcification (CAC score 100 to 399), 30 (23\%) had severe calcification (CAC score 400 to 999), while $17(13 \%)$ patients had extensive calcifications (CAC score $\geq 1000$ ).

\section{CT Myocardial Perfusion}

A total of 55 (42\%) patients had myocardial perfusion abnormalities at stress, $6(5 \%)$ patients had only perfusion defects at rest and $49(37 \%)$ patients had 1 or more segments with reversible defects on CTP, indicating inducible myocardial ischemia. The mean (range) SRS, SSS, and SDS were 0.22 (0 to 12 ), 2.26 (0 to 17), and 2.04 (0 to 16), respectively. The median effective radiation dose for CTP was $2.8 \mathrm{mSv}$ (IQR 1.8 to 4.4).

\section{Relation Between CAC Score and Inducible Myocardial Ischemia on CTP}

In the subgroups CAC score 1 to $99, \mathrm{CAC}$ score 100 to 399, CAC score 400 to 999 , and CAC score $\geq 1000$ 
Table 1. Clinical characteristics divided according to the presence of inducible myocardial ischemia

\begin{tabular}{|c|c|c|c|c|}
\hline & $\begin{array}{c}\text { Total } \\
\text { population } \\
(\mathbf{n}=131)\end{array}$ & $\begin{array}{c}\text { Myocardial } \\
\text { ischemia } \\
(n=49)\end{array}$ & $\begin{array}{l}\text { No myocardial } \\
\text { ischemia } \\
(\mathbf{n}=\mathbf{8 2})\end{array}$ & $P$ value \\
\hline Age (years) & $62 \pm 9.4$ & $63 \pm 7.9$ & $61 \pm 10.2$ & 0.332 \\
\hline Male, $n(\%)$ & $73(56)$ & $27(55)$ & $46(56)$ & 0.912 \\
\hline BMI $\left(\mathrm{kg} / \mathrm{m}^{2}\right)$ & $27 \pm 4$ & $26 \pm 4$ & $27 \pm 5$ & 0.449 \\
\hline \multicolumn{5}{|l|}{ Cardiovascular risk factors } \\
\hline Current smoking, n (\%) & $18(14)$ & $9(18)$ & $9(11)$ & 0.296 \\
\hline Diabetes, n (\%) & $32(24)$ & $11(22)$ & $21(26)$ & 0.684 \\
\hline Family history of CVD, $\mathrm{n}(\%)$ & $67(51)$ & $27(55)$ & $40(49)$ & 0.484 \\
\hline Hypercholesterolemia, n (\%) & $57(44)$ & $24(49)$ & $33(40)$ & 0.329 \\
\hline Hypertension, n (\%) & $80(61)$ & $35(71)$ & $45(55)$ & 0.060 \\
\hline \multicolumn{5}{|l|}{ Updated Diamond-Forrester risk score (\%) } \\
\hline Intermediate $(20-80 \%)$ pre-test risk, $\mathrm{n}(\%)$ & $84(64)$ & $34(69)$ & $50(61)$ & 0.376 \\
\hline \multicolumn{5}{|l|}{ Medication } \\
\hline Aspirin, n (\%) & $35(27)$ & $14(29)$ & $21(26)$ & 0.711 \\
\hline Thienopyridine, n (\%) & $3(2)$ & $2(4)$ & $1(1)$ & 0.556 \\
\hline OAC, $n(\%)$ & $14(11)$ & $6(12)$ & $8(10)$ & 0.772 \\
\hline$\beta$-blocker, n (\%) & $70(53)$ & $30(61)$ & $40(49)$ & 0.167 \\
\hline Statin, $n(\%)$ & $61(47)$ & $24(49)$ & $37(45)$ & 0.668 \\
\hline Diuretic, n (\%) & $26(20)$ & $10(20)$ & $16(20)$ & 0.901 \\
\hline ACE-I/ARB & $55(42)$ & $23(47)$ & $32(39)$ & 0.374 \\
\hline
\end{tabular}

Values are shown as $\mathrm{n}(\%)$ or as mean \pm standard deviation

$A C E-I$, angiotensin-converting enzyme inhibitor; $A R B$, angiotensin receptor blocker; $B M I$ body mass index; $C A B G$, coronary artery bypass grafting; $C V D$, cardiovascular disease; $O A C$, oral anticoagulants; $P C I$, percutaneous coronary intervention

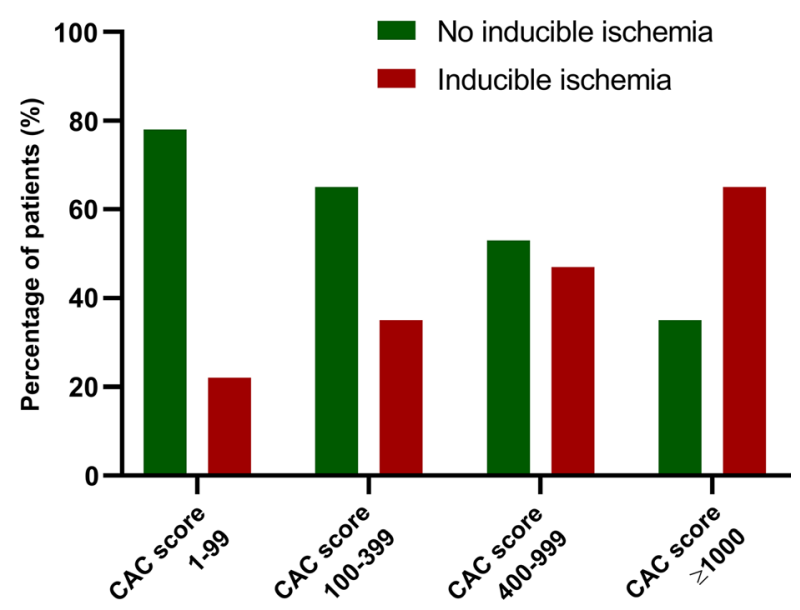

Figure 1. Relationship between percentage of patients with inducible myocardial ischemia and CAC score subgroups. The percentage of subjects with inducible myocardial ischemia on CTP increased with increasing CAC score severity. CAC score, coronary artery calcium score. evidence of inducible myocardial ischemia on CTP was seen in $9(22 \%), 15(35 \%), 14(47 \%)$, and $11(65 \%)$, respectively. (Figure 1) The relation between CAC score and inducible myocardial ischemia per coronary artery is shown in Table 2. The LAD had higher CAC score independent of the presence of inducible myocardial ischemia or not. The extent of inducible myocardial ischemia (SDS) related to the predefined CAC score subgroups is shown in Figure 2 and the per vessel analysis is shown in Table 3. There was a moderate but significant positive correlation between CAC score and SDS $(r=0.368 ; P<0.0001)$.

\section{Uni- and Multivariable Analysis for Inducible Myocardial Ischemia on CTP}

The uni- and multivariable analysis for the presence of inducible myocardial ischemia on CTP are shown in Table 4. No cardiovascular risk factors were significantly correlated with inducible myocardial ischemia (Table 3). CAC score was significantly correlated with 
Table 2. CAC score divided according to the presence of inducible myocardial ischemia

\begin{tabular}{lcccc}
\hline & $\begin{array}{c}\text { Total population } \\
(\mathbf{n}=\mathbf{1 3 1})\end{array}$ & $\begin{array}{c}\text { Myocardial ischemia } \\
(\mathbf{n}=\mathbf{4 9})\end{array}$ & $\begin{array}{c}\text { No myocardial } \\
\text { ischemia (n= 82) }\end{array}$ & P value \\
\hline CAC score & $241(73-539)$ & $438(189-905)$ & $167(40-421)$ & $0.001^{*}$ \\
Per vessel & & & & \\
CAC score LAD & $119(30-280)$ & $216(94-415)$ & $84(21-228)$ & $0.001^{*}$ \\
CAC score RCA & $24(0-124)$ & $71(4-216)$ & $11(0-84)$ & $0.005^{*}$ \\
CAC score LCX & $9(0-101)$ & $34(1-186)$ & $6.5(0-73)$ & $0.019^{*}$
\end{tabular}

Values are presented as median (25th-75th percentile)

$C A C$ score, coronary artery calcium score; $L A D$, left anterior descending coronary artery; $L C X$, left circumflex coronary artery; $R C A$, right coronary artery

$* P<0.05$

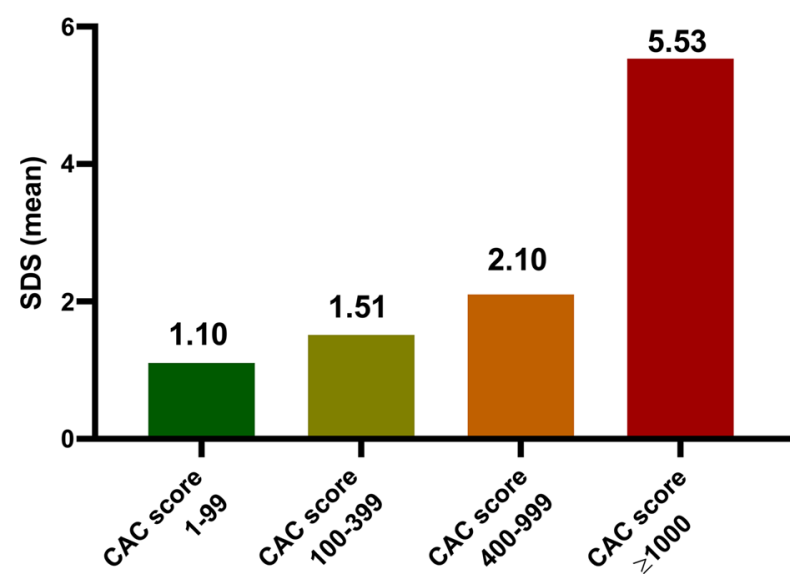

Figure 2. Relationship between extent of inducible myocardial ischemia on CTP and CAC score subgroups. A significant difference was found for the extent of inducible myocardial ischemia between the CAC score subgroups $(P=0.002)$. CAC score, coronary artery calcium score; $S D S$, summed difference score.

inducible myocardial ischemia on CTP in univariable analysis (OR 1.001; 95\% CI 1.000 to $1.001 ; P=0.013$ ) and remained significant in the multivariable analysis (OR: 1.001 per 1 Agatston Unit; 95\% CI 1.000 to 1.001; $P=0.029)$.

\section{DISCUSSION}

This study is the first to describe the relationship between CAC score and the presence of inducible myocardial ischemia on CTP. We observed a positive correlation between the burden of CAC and inducible myocardial ischemia on CTP. The frequency of inducible myocardial ischemia on CTP was three times higher for patients with extensive calcification compared to patients with mild calcifications. Moreover, the majority of symptomatic patients referred for CAC scoring with extensive calcifications had inducible myocardial ischemia on CTP.

\section{CAC Score}

Several large retrospective and prospective studies have shown the prognostic value of CAC score measured by coronary CT in asymptomatic patients and its value to improve risk detection over traditional risk factors. ${ }^{3-9}$ The prognostic value of CAC score has also been demonstrated in symptomatic patients. ${ }^{10-14}$

\section{CAC Score and CTP}

Previous studies applying both CAC score and CTP have primarily focused on the incremental diagnostic value of CTP in the setting of decreased interpretability of coronary CTA in patients with high CAC score. ${ }^{19,20}$ Sharma et al. investigated the diagnostic performance of CTP and coronary CTA in 381 patients with intermediate and high risk for CAD and patients with known $\mathrm{CAD}^{20}{ }^{20}$ The population was divided in patients with a CAC score 1 to 399 and CAC score $\geq 400$. In patients with an CAC score $\geq 400$ combined use of coronary CTA and CTP showed superior diagnostic accuracy than coronary CTA or CTP alone, using stenosis $\geq 50 \%$ on invasive coronary angiography with corresponding stress perfusion defect on SPECT-MPI as a reference standard. Ladeiras-Lopes et al., reached the same conclusions in a cohort of 95 symptomatic patients with an intermediate pre-test probability of CAD using invasive fractional flow reserve (FFR) as a gold standard. ${ }^{19}$ The direct relationship between CAC score and the presence of inducible myocardial ischemia on CTP has not been investigated before. 
Table 3. SDS per coronary artery according to the CAC score subgroups

\begin{tabular}{lccccc}
\hline & $\begin{array}{c}\text { CAC score 1-99 } \\
(\mathbf{n}=\mathbf{4 1})\end{array}$ & $\begin{array}{c}\text { CAC score } \\
\mathbf{1 0 0 - 3 9 9}(\mathbf{n}=\mathbf{4 3 )}\end{array}$ & $\begin{array}{c}\text { CAC score } \\
\mathbf{4 0 0 - 9 9 9}(\mathbf{n}=\mathbf{3 0})\end{array}$ & $\begin{array}{c}\text { CAC score } \\
\mathbf{1 0 0 0}(\mathbf{1 0 0}=\mathbf{1 7})\end{array}$ & $\boldsymbol{P}$ value \\
\hline SDS LAD & $0.63(0-12)$ & $0.67(0-7)$ & $1.07(0-6)$ & $3.82(0-15)$ & $<0.001^{*}$ \\
SDS RCA & $0.10(0-2)$ & $0.42(0-5)$ & $0.43(0-2)$ & $1.18(0-6)$ & $0.013^{*}$ \\
SDS LCX & $0.37(0-6)$ & $0.42(0-4)$ & $0.60(0-6)$ & $0.53(0-4)$ & 0.672
\end{tabular}

Values are presented as mean (minimum-maximum)

$C A C$ score, coronary artery calcium score; $L A D$, left anterior descending coronary artery; $L C X$, left circumflex coronary artery; $R C A$, right coronary artery; $S D S$, summed difference score

$* P<0.05$

Table 4. Uni- and multivariable analysis for inducible myocardial ischemia

\begin{tabular}{lcccc}
\hline & $\begin{array}{c}\text { Univariable odds } \\
\text { ratio (95\% CI) }\end{array}$ & P value & $\begin{array}{c}\text { Multivariable Odds } \\
\text { ratio (95\% CI) }\end{array}$ & P value \\
\hline Age & $1.018(0.980-1.058)$ & 0.361 & $1.000(0.958-1.045)$ & 0.985 \\
Male & $0.960(0.471-1.958)$ & 0.912 & $0.800(0.350-1.829)$ & 0.597 \\
BMI & $0.968(0.891-1.052)$ & 0.447 & & \\
Current smoking & $1.825(0.671-4.967)$ & 0.239 & & \\
Diabetes & $0.841(0.365-1.937)$ & 0.684 & & \\
Family history of CVD & $1.289(0.633-2.622)$ & 0.484 & & \\
Hypercholesterolemia & $1.425(0.699-2.908)$ & 0.330 & & \\
Hypertension & $2.056(0.964-4.383)$ & 0.062 & $1.626(0.731-3.618)$ & 0.234 \\
CAC score & $1.001(1.000-1.001)$ & 0.013 & $1.001(1.000-1.001)$ & $0.029^{*}$
\end{tabular}

$C A C$ score, coronary artery calcium score; $C V D$, cardiovascular disease

${ }^{*} P<0.05$

\section{CAC Score and SPECT-MPI}

The relationship between $\mathrm{CAC}$ and myocardial ischemia on SPECT-MPI has been investigated previously. He et al., showed in a population of 411 predominantly asymptomatic patients that CAC severity was the strongest predictor for the presence of silent myocardial ischemia on SPECT-MPI. ${ }^{15}$ Several studies have shown similar results with increasing rates of myocardial ischemia on SPECT-MPI and PET-MPI with increasing CAC score subgroup. ${ }^{16,17} \mathrm{~A}$ meta-analysis from Bavishi et al., including 20 studies showed a wide range of prevalence of inducible myocardial ischemia on SPECT-MPI or PET-MPI, among the different studies but a consistent increase of inducible myocardial ischemia with increasing CAC score subgroup. ${ }^{16}$ In a large a cohort of 4897 symptomatic patients with lowto-intermediate risk, Engbers et al. showed that CAC score was an independent predictor for abnormal SPECT-MPI. ${ }^{17}$ The frequency of abnormal SPECT-
MPI increased with higher CAC score, from 19\% in patients with mild calcifications to $50 \%$ in patients with extensive calcifications, similar to the results in our study. Interestingly, Engbers et al. also showed that combined evaluation of SPECT-MPI and CAC score provided incremental prognostic information over the individual modalities. ${ }^{17}$ This was also shown by Nappi et al. in a population of 156 patients. ${ }^{27}$ Chang et al. reached the same conclusions in 1126 mostly asymptomatic subjects. ${ }^{28}$ Moreover, in a study by Assante et al., the combined evaluation of CAC score and coronary vascular function as assessed by coronary flow reserve also provided incremental risk stratification for the prediction of adverse cardiac events. ${ }^{29}$

\section{CAC Score and Other Functional Tests}

The relation between CAC score and other functional tests has also been investigated. Ramakrishna et al., investigated the relationship between CAC score 
and exercise echocardiography in a population of 556 patients. $^{30}$ The correlation between CAC score and exercise wall motion score index (WMSI) was significant, but limited $(r=0.17)$, underscoring the difference between anatomical and functional testing. Patients with both CAC score $>100$ and also exercise WMSI $>1$ were 4 times more likely to experience a myocardial infarction or die during a follow-up of 5 years compared to patients with a CAC score $\leq 100$ and a normal exercise WMSI. Janssen et al., investigated the relation between CAC score and dobutamine cardiovascular magnetic resonance imaging (CMR). ${ }^{31}$ They showed that in a population of 114 symptomatic patients a CAC score $\leq 100$ had a negative and positive predictive value of 0.96 and 0.29 , respectively, for predicting inducible myocardial ischemia during dobutamine CMR.

CTP has several benefits over SPECT-MPI and other stress testing modalities investigating myocardial ischemia. CTP allows for a fast and simultaneous assessment of anatomical and functional parameters in one session. Furthermore, a recent meta-analysis by Takx et al., showed CTP to accurately rule out hemodynamic significant CAD using invasive FFR as a golden standard, whereas SPECT-MPI and echocardiography were less accurate. ${ }^{32}$

\section{LIMITATIONS}

This study has several limitations, inherent to its retrospective and single-center design. The exclusion of patients with uninterpretable CTP imaging might have introduced selection bias. We analyzed the CAC score using the Agatston method and did not incorporate the distribution of the calcifications in the coronary vessel. Although inducible myocardial ischemia has shown prognostic value, in our study we did not look at clinical endpoints.

\section{CONCLUSIONS}

In a population of symptomatic patients, the majority of patients with extensive calcification had evidence of inducible myocardial ischemia on CTP. CAC score was an independent predictor of inducible myocardial ischemia on CTP.

\section{NEW KNOWLEDGE GAINED}

To the best of our knowledge this is the first study to investigate the direct relationship between CAC score and inducible myocardial ischemia on CTP. Our results suggest that the majority of stable chest pain patients with extensive CAC score have evidence of inducible ischemia on CTP.

\section{Disclosures}

The department of Cardiology of the Leiden University Medical Centre received research Grants from Biotronik, Medtronic, Boston Scientific, GE Healthcare, and Edwards Lifesciences. Arthur Scholte received speakers fee from Canon Medical Systems and GE Healthcare. This research did not receive any specific Grant from funding agencies in the public, commercial, or not-for-profit sectors.

\section{Open Access}

This article is distributed under the terms of the Creative Commons Attribution 4.0 International License (http://creativecommons.org/licenses/by/4.0/), which permits unrestricted use, distribution, and reproduction in any medium, provided you give appropriate credit to the original author(s) and the source, provide a link to the Creative Commons license, and indicate if changes were made.

\section{References}

1. Agatston AS, Janowitz WR, Hildner FJ, Zusmer NR, Viamonte M Jr, Detrano R. Quantification of coronary artery calcium using ultrafast computed tomography. J Am Coll Cardiol 1990;15:827-32.

2. Sangiorgi G, Rumberger JA, Severson A, Edwards WD, Gregoire $\mathrm{J}$, Fitzpatrick LA, et al. Arterial calcification and not lumen stenosis is highly correlated with atherosclerotic plaque burden in humans: a histologic study of 723 coronary artery segments using nondecalcifying methodology. J Am Coll Cardiol 1998;31:126-33.

3. Greenland P, LaBree L, Azen SP, Doherty TM, Detrano RC. Coronary artery calcium score combined with Framingham score for risk prediction in asymptomatic individuals. JAMA 2004;291:210-5.

4. Arad Y, Goodman KJ, Roth M, Newstein D, Guerci AD. Coronary calcification, coronary disease risk factors, C-reactive protein, and atherosclerotic cardiovascular disease events: The St Francis Heart Study. J Am Coll Cardiol 2005;46:158-65.

5. Vliegenthart R, Oudkerk M, Hofman A, Oei HH, van Dijck W, van Rooij FJ, et al. Coronary calcification improves cardiovascular risk prediction in the elderly. Circulation 2005;112:572-7.

6. Budoff MJ, Shaw LJ, Liu ST, Weinstein SR, Mosler TP, Tseng $\mathrm{PH}$, et al. Long-term prognosis associated with coronary calcification: Observations from a registry of 25,253 patients. J Am Coll Cardiol 2007;49:1860-70.

7. Detrano R, Guerci AD, Carr JJ, Bild DE, Burke G, Folsom AR, et al. Coronary calcium as a predictor of coronary events in four racial or ethnic groups. N Engl J Med 2008;358:1336-45.

8. Elias-Smale SE, Proenca RV, Koller MT, Kavousi M, van Rooij FJ, Hunink MG, et al. Coronary calcium score improves classification of coronary heart disease risk in the elderly: The Rotterdam study. J Am Coll Cardiol 2010;56:1407-14.

9. Geisel MH, Bauer M, Hennig F, Hoffmann B, Lehmann N, Mohlenkamp S, et al. Comparison of coronary artery calcification, carotid intima-media thickness and ankle-brachial index for predicting 10-year incident cardiovascular events in the general population. Eur Heart J 2017;38:1815-22.

10. Detrano R, Hsiai T, Wang S, Puentes G, Fallavollita J, Shields P, et al. Prognostic value of coronary calcification and angiographic stenoses in patients undergoing coronary angiography. J Am Coll Cardiol 1996;27:285-90. 
11. Keelan PC, Bielak LF, Ashai K, Jamjoum LS, Denktas AE, Rumberger JA, et al. Long-term prognostic value of coronary calcification detected by electron-beam computed tomography in patients undergoing coronary angiography. Circulation 2001;104:412-7.

12. Schmermund A, Stang A, Mohlenkamp S, Eggebrecht H, Baumgart D, Gilbert V, et al. Prognostic value of electron-beam computed tomography-derived coronary calcium scores compared with clinical parameters in patients evaluated for coronary artery disease Prognostic value of EBCT in symptomatic patients. Z. Kardiol 2004;93:696-705.

13. Sarwar A, Shaw LJ, Shapiro MD, Blankstein R, Hoffmann U, Cury RC, et al. Diagnostic and prognostic value of absence of coronary artery calcification. JACC Cardiovasc Imaging 2009;2:675-88.

14. Petretta M, Daniele S, Acampa W, Imbriaco M, Pellegrino T, Messalli G, et al. Prognostic value of coronary artery calcium score and coronary $\mathrm{CT}$ angiography in patients with intermediate risk of coronary artery disease. Int $\mathrm{J}$ Cardiovasc Imaging 2012;28:1547-56.

15. He ZX, Hedrick TD, Pratt CM, Verani MS, Aquino V, Roberts R, et al. Severity of coronary artery calcification by electron beam computed tomography predicts silent myocardial ischemia. Circulation 2000;101:244-51.

16. Bavishi C, Argulian E, Chatterjee S, Rozanski A. CACS and the frequency of stress-induced myocardial ischemia during MPI: A meta-analysis. JACC Cardiovasc Imaging 2016;9:580-9.

17. Engbers EM, Timmer JR, Ottervanger JP, Mouden M, Knollema S, Jager PL. Prognostic value of coronary artery calcium scoring in addition to single-photon emission computed tomographic myocardial perfusion imaging in symptomatic patients. Circ Cardiovasc Imaging 2016;9:003966.

18. George RT, Mehra VC, Chen MY, Kitagawa K, Arbab-Zadeh A, Miller JM, et al. Myocardial CT perfusion imaging and SPECT for the diagnosis of coronary artery disease: A head-to-head comparison from the CORE320 multicenter diagnostic performance study. Radiology 2014;272:407-16.

19. Ladeiras-Lopes R, Bettencourt N, Ferreira N, Sampaio F, PiresMorais G, Santos L, et al. CT myocardial perfusion and coronary CT angiography: Influence of coronary calcium on a stress-rest protocol. J Cardiovasc Comput Tomogr 2016;10:215-20.

20. Sharma RK, Arbab-Zadeh A, Kishi S, Chen MY, Magalhaes TA, George RT, et al. Incremental diagnostic accuracy of computed tomography myocardial perfusion imaging over coronary angiography stratified by pre-test probability of coronary artery disease and severity of coronary artery calcification: The CORE320 study. Int J Cardiol 2015;201:570-7.

21. Genders TSS, Steyerberg EW, Alkadhi H, Leschka S, Desbiolles L, Nieman K, et al. A clinical prediction rule for the diagnosis of coronary artery disease: Validation, updating, and extension. Eur Heart J 2011;32:1316-30.

22. van Rosendael AR, Kroft LJ, Broersen A, Dijkstra J, van den Hoogen IJ, van Zwet EW, et al. Relation between quantitative coronary CTA and myocardial ischemia by adenosine stress myocardial CT perfusion. J Nucl Cardiol 2017;24:1253-62.

23. van Rosendael AR, de Graaf MA, Dimitriu-Leen AC, van Zwet EW, van den Hoogen IJ, Kharbanda RK, et al. The influence of clinical and acquisition parameters on the interpretability of adenosine stress myocardial computed tomography perfusion. Eur Heart J Cardiovasc Imaging. 2017;18:203-11.

24. Hausleiter J, Meyer T, Hermann F, Hadamitzky M, Krebs M, Gerber TC, et al. Estimated radiation dose associated with cardiac CT angiography. JAMA 2009;301:500-7.

25. Cerqueira MD, Weissman NJ, Dilsizian V, Jacobs AK, Kaul S, Laskey WK, et al. Standardized myocardial segmentation and nomenclature for tomographic imaging of the heart. A statement for healthcare professionals from the Cardiac Imaging Committee of the Council on Clinical Cardiology of the American Heart Association. Circulation 2002;105:539-42.

26. Mehra VC, Valdiviezo C, Arbab-Zadeh A, Ko BS, Seneviratne SK, Cerci R, et al. A stepwise approach to the visual interpretation of CT-based myocardial perfusion. J Cardiovasc Comput Tomogr 2011;5:357-69.

27. Nappi C, Nicolai E, Daniele S, Acampa W, Gaudieri V, Assante R, et al. Long-term prognostic value of coronary artery calcium scanning, coronary computed tomographic angiography and stress myocardial perfusion imaging in patients with suspected coronary artery disease. J Nucl Cardiol 2018;25:833-41.

28. Chang SM, Nabi F, Xu J, Peterson LE, Achari A, Pratt CM, et al. The coronary artery calcium score and stress myocardial perfusion imaging provide independent and complementary prediction of cardiac risk. J Am Coll Cardiol 2009;54:1872-82.

29. Assante R, Acampa W, Zampella E, Arumugam P, Nappi C, Gaudieri V, et al. Prognostic value of atherosclerotic burden and coronary vascular function in patients with suspected coronary artery disease. Eur J Nucl Med Mol Imaging 2017;44:2290-8.

30. Ramakrishna G, Breen JF, Mulvagh SL, McCully RB, Pellikka PA. Relationship between coronary artery calcification detected by electron-beam computed tomography and abnormal stress echocardiography: Association and prognostic implications. J Am Coll Cardiol 2006;48:2125-31.

31. Janssen CH, Kuijpers D, Vliegenthart R, Overbosch J, van Dijkman PR, Zijlstra F, et al. Coronary artery calcification score by multislice computed tomography predicts the outcome of dobutamine cardiovascular magnetic resonance imaging. Eur Radiol 2005; $15: 1128-34$

32. Takx RA, Blomberg BA, El Aidi H, Habets J, de Jong PA, Nagel E, et al. Diagnostic accuracy of stress myocardial perfusion imaging compared to invasive coronary angiography with fractional flow reserve meta-analysis. Circ Cardiovasc Imaging 2015;8:e02666.

Publisher's Note Springer Nature remains neutral with regard to jurisdictional claims in published maps and institutional affiliations. 